\title{
MATRIX KERNELS FOR MEASURES ON PARTITIONS
}

\author{
EUGENE STRAHOV
}

\begin{abstract}
We consider the problem of computation of the correlation functions for the $z$-measures with the deformation (Jack) parameters 2 or $1 / 2$. Such measures on partitions are originated from the representation theory of the infinite symmetric group, and in many ways are similar to the ensembles of Random Matrix Theory of $\beta=4$ or $\beta=1$ symmetry types. For a certain class of such measures we show that correlation functions can be represented as Pfaffians including $2 \times 2$ matrix valued kernels, and compute these kernels explicitly. We also give contour integral representations for correlation kernels of closely connected measures on partitions.
\end{abstract}

\section{INTRODUCTION}

1.1. Preliminaries and formulation of the problem. It was observed by many authors that number of problems of statistical mechanics and of combinatorial probability can be reduced to investigation of random Young diagrams. In particular, increasing subsequences problems, certain last-passage percolation and growth models, random tilings are all examples of such a situation, see Baik, Deift, and Johansson [1], Baik and Rains [2, 3, 4, Johansson [22, 23, 24, Prähover and Spohn [38, Ferrari and Prähover [19], and references therein.

For instance, in the analysis of the polynuclear growth (PNG) model in one spatial dimension the relation with measures on Young diagrams arises as follows, see Ferrari and Prähover for a recent review [19]. Following Ferrari and Prähover, let us describe the growing surface at time $t$ by an integer-valued height function $x \rightarrow h(x, t) \in \mathbb{Z}$. For fixed time $t \in \mathbb{R}$, one considers the height profile $x \rightarrow h(x, t)$. The height $h$, as $x$ increases, has jumps of height one at the discontinuity points. By definition, the dynamics of the model has a deterministic and a

Date: April 7, 2008.

Key words and phrases. Random partitions, symmetric functions, random Young diagrams, correlation functions, Pfaffian point processes.

Department of Mathematics, The Hebrew University of Jerusalem, Givat Ram, Jerusalem 91904. E-mail: strahov@math.huji.ac.il. Supported by US-Israel Binational Science Foundation (BSF) Grant No. 2006333. 
stochastic part. The deterministic part is described by the condition that the up-steps move to the left with unit speed, and the down steps move to the right with unit speed. When a pair of up-and down steps collide, they disappear. The stochastic parts describe nucleation events (creation of up-down steps on the surface). It is assumed that once a pair of up-down steps is created, it follows the deterministic dynamics. The main problem in this context is to describe the fluctuations of height profile $h(x, t)$ as time grows. Typically one considers two cases of particular interest, called the PNG droplet and the flat PNG. In both cases the investigation of $h(x, t)$ at point $x=0$ is reduced to that of the first row of a random Young diagram. In the first situation (the PNG droplet) the relevant measure on Young diagrams is the Poissonized Plancherel measure, and in the second situation (the flat PNG) the relevant measure on Young diagrams can be understood as the Poissonized Plancherel measure with Jack parameter $1 / 2$. Note also that the Plancherel measures with Jack parameters 2, 1/2 also appear in the analysis of the symmetrized increasing subsequence problems, see Baik and Rains [3], Forrester, Nagao and Rains [21].

The Plancherel measures mentioned above can be understood as limits of the $z$-measures $\mathcal{M}_{z, z^{\prime}, \theta, \xi}$ which are probability measures on the set $\mathbb{Y}$ of all Young diagrams. These measures arise in the context of the representation theory of the infinite symmetric group. Namely, let $S(\infty)$ be the infinite symmetric group whose elements are finite permutations of $\{1,2, \ldots\}$. Kerov, Olshanski, and Vershik [28] (see also a review paper by Olshanski [37]) constructed a family $\left\{T_{z}: z \in \mathbb{C}\right\}$ of unitary representations of two copies $G$ of $S(\infty)$. Such representations were called the generalized regular representations of the infinite symmetric group. Each representation $T_{z}$ with $z \neq 0$ can be realized in the Hilbert space $L^{2}\left(\mathfrak{S}, \mu_{t}\right)$, where $\mathfrak{S}$ is a compatification of $S(\infty)$ (called the space of virtual permutations), $\left\{\mu_{t}\right\}$ is a family of $G$ quasiinvariant measures on virtual permutations, and $t=|z|^{2}$. Denote by $\chi^{z}$ the character of $T_{z}$. According to Kerov, Olshanski and Vershik [28], $\chi^{z}$ is a central positive definite function on $S(\infty)$ such that, for any $n=1,2, \ldots$, its restriction to $S(n)$ is

$$
\chi_{n}^{z}=\sum_{\lambda \in \mathbb{Y}_{n}} \mathcal{M}_{z, \bar{z}}^{(n)}(\lambda) \frac{\chi^{\lambda}}{\chi^{\lambda}(e)} .
$$

Here $\chi^{\lambda}$ denotes the irreducible character of $S(n)$ parameterized by the Young diagram $\lambda$, and $\mathbb{Y}_{n}$ is the set of all Young diagrams with $n$ boxes. It can be shown that for every $n=1,2, \ldots \mathcal{M}_{z, \bar{z}}^{(n)}$ is a probability distribution on the set $\mathbb{Y}_{n}$. 
Borodin and Olshanski [6, 8, 9] considered a 3-parameter family of measures $\mathcal{M}_{z, z^{\prime}, \xi}$ (called $z$-measures) on the set of all Young diagrams $\mathbb{Y}$. This family was constructed by mixing of $\mathcal{M}_{z, z^{\prime}}^{(n)}$, see Borodin and Olshanski [6] for details. Under certain conditions on parameters, $\mathcal{M}_{z, z^{\prime}, \xi}$ is a probability measure on $\mathbb{Y}$. Borodin and Olshanski [6] showed that $\mathcal{M}_{z, z^{\prime}, \xi}$ defines a determinantal point process, and that $\mathcal{M}_{z, z^{\prime}, \xi}$ can be studied on the same level as the Gaussian Unitary Ensemble of Random Matrix Theory (see, for example, Mehta [30], Deift [17], Forrester [20] as basic references on ensembles of random matrices; for a review of determinantal point processes see Soshnikov [41]).

As it follows from Kerov [27], Borodin and Olshanski [10] it is natural to consider a deformation $\mathcal{M}_{z, z^{\prime}, \theta, \xi}$ of $\mathcal{M}_{z, z^{\prime}, \xi}$, where $\theta>0$ is called the parameter of deformation (or the Jack parameter). As it shown in Borodin and Olshanski [10], $\mathcal{M}_{z, z^{\prime}, \theta, \xi}$ are in many ways similar to loggas (random-matrix) models with arbitrary $\beta=2 \theta$. In particular, if $\theta=2$ or $\theta=1 / 2$ one expects that $\mathcal{M}_{z, z^{\prime}, \theta, \xi}$ will lead to Pfaffian point processes, similar to ensembles of Random Matrix Theory of $\beta=4$ or $\beta=1$ symmetry types. Thus, it is a natural problem to compute the correlation functions for $z$-measures with the deformation parameters $\theta=2$ or $1 / 2$.

More explicitly, set

$$
\mathcal{D}_{2}(\lambda)=\left\{\lambda_{i}-2 i\right\}
$$

That is, $\mathcal{D}_{2}(\lambda)$ is an infinite subset of $\mathbb{Z}$ corresponding to the Young diagram $\lambda$, and containing infinitely many integers. Let $X=\left(x_{1}, \ldots, x_{n}\right)$ be a subset of $\mathbb{Z}$ consisting of $n$ distinct points, and define

$$
\varrho_{z, z^{\prime}, 2, \xi}(X)=\mathcal{M}_{z, z^{\prime}, 2, \xi}\left(\left\{\lambda \mid X \subset \mathcal{D}_{2}(\lambda)\right\}\right) \text {. }
$$

The problem is to give an explicit formula for $\varrho_{z, z^{\prime}, 2, \xi}(X)$, i.e. to compute the correlation function of $\mathcal{M}_{z, z^{\prime}, 2, \xi}$. Note that as soon as $\mathcal{M}_{z, z^{\prime}, \theta, \xi}$ and $\mathcal{M}_{z, z^{\prime}, 1 / \theta, \xi}$ are related by

$$
\mathcal{M}_{z, z^{\prime}, \theta, \xi}(\lambda)=\mathcal{M}_{-z / \theta,-z^{\prime} / \theta, 1 / \theta, \xi}\left(\lambda^{\prime}\right),
$$

it is enough to consider the $z$-measures with the deformation parameter $\theta=2$. Defining $\varrho_{z, z^{\prime}, 2, \xi}$ as above we do not require positivity of $\mathcal{M}_{z, z^{\prime}, 2, \xi}$.

\subsection{Summary of results.}

1.2.1. A formula for the correlation function of $\mathcal{M}_{z, z-1,2, \xi}$. Theorem 3.1 gives an explicit formula for the correlation function of $\mathcal{M}_{z, z-1,2, \xi}$. This result shows that the correlation function of $\mathcal{M}_{z, z-1,2, \xi}$ can be written as a Pfaffian of a $2 \times 2$ matrix valued kernel. The entries

of this kernel are expressible in terms of one function, $S_{z, z-1,2, \xi}(x, y)$, 
which admits a contour integral representation, see Theorem 3.1, a). Theorem 3.1, b) gives a formula for $S_{z, z-1,2, \xi}(x, y)$ in terms of the Gauss hypergeometric functions.

Thus, Theorem 3.1 provides a partial solution of the problem stated in Section 1.1, and it is the main result of the present paper.

1.2.2. A contour integral representation for correlation functions of Pfaffian Schur measures. In fact, results of Theorem 3.1 can be obtained from a more general algebraic fact. We consider measures on partitions which can be understood as Pfaffian analogues of Okounkov's Schur measures, and derive contour integral representations for correlation kernels in Theorem 4.1. Then Theorem 3.1 is obtained by considering a specific specialization of the algebra of symmetric functions. As it will be evident from the proof of Theorem [3.1, the correlation function of $\mathcal{M}_{z, z^{\prime}, 2, \xi}$ in the case of arbitrary parameters $z, z^{\prime}$ cannot be deduced from Theorem 4.1, and a different approach is needed.

1.2.3. Correlation functions for the Plancherel measure with the deformation parameter $\theta=2$. When both $z, z^{\prime}$ go to infinity, the $z$-measures with general parameter $\theta>0$ have limits. These limits can be understood as Plancherel measures with general parameter $\theta>0$. Theorem 7.1, a) gives the contour integral representation for the correlation kernel of the Plancherel measure with the deformation parameter $\theta=2$, and Theorem [7.1, b) gives a formula for these correlation kernels in terms of Bessel functions.

\subsection{Remarks on related works.}

1.3.1. It is known that a number of important measures on partitions lead to determinantal point processes. The most well-known example of measures of this type is the poissonization of the Plancherel measure, which was considered by many researches, see Baik, Deift and Johansson [1], Borodin, Okounkov and Olshanski [7], the review articles by Deift [16, and by van Moerbeke [31. The z-measures (with $\theta=1$ ) is another family of measures leading to a determinantal point process. The fact that $z$-measures define a determinantal process was first proved by Borodin and Olshanski [6]. The paper by Borodin and Olshanski [6] contains an explicit computation of the correlation kernel, and shows that the correlation kernel can be expressed through Gauss hypergeometric functions. The $z$-measures on partitions were also studied in Borodin and Olshanski [8, 9], Okounkov [35], Borodin, Olshanski, and Strahov [12]. Note that for special values of parameters $z$-measures turn into discrete orthogonal polynomial ensembles. 
Such ensembles are related with different exactly solvable probabilistic models, see Borodin and Olshanski [8], and the references therein.

1.3.2. Okounkov 32] introduced a family of measures on partitions called the Schur measures. This family of measures includes the $z$ measures (with $\theta=1$ ) and the Poissonized Plancherel measure as special cases. Okounkov [33, 34] discusses different applications of such measures. For relations of the Schur measures with the space of free fermions see Okounkov [32], a recent review article by Harnard and Orlov [26], and the references therein.

1.3.3. The Schur processes introduced in Okounkov and Reshetikhin [36], and the periodic Schur measures introduced in Borodin [5] are generalizations of the Schur measures, which also define determinantal point processes. The correlation kernels of the Schur measures, of the Schur processes and of the periodic Schur processes have contour integral representations. As it was demonstrated in Okounkov [33, 34], Okounkov and Reshetikhin [36], Borodin [5] the contour integral representations for the correlation kernels have many advantages, in particular, such representations are very convenient for the asymptotic analysis.

1.3.4. The class of measures considered in Section 4 of the present paper was first introduced by Rains [39] in the context of symmetrized increasing subsequence problems, and in certain sense these measures are Pfaffian analogues of Okounkov's Schur measures. Paper by Rains [39] also contains the idea of computation of the correlation kernels. Borodin and Rains [15], Vuletić [43] have considered different classes of measures (the Pfaffian Schur processes in Borodin and Rains [15], and the shifted Schur processes in Vuletić [43]) whose correlation functions are expressible as Pfaffians of matrix valued kernels. It can be verified that the results of Theorem 4.1 can be deduced from that of Theorem 3.3 in Borodin and Rains [15]. However, the author of this paper was not able to find the explicit formula for $S_{\text {Schur,2 }}(x, y)$ (see Theorem 4.1) in the literature. In the present paper we give an independent proof of Theorem 4.1 .

1.3.5. The correlation functions for measures, which are in many ways similar to the Plancherel measures with the deformation parameters $\theta=1 / 2,2$, were previously computed by different authors, see Ferrari [18, Lemma 5.2, Sasamoto and Imamura [40, and Forrester, Nagao, 
and Rains [21], Section 3. In particular, Ferarri [18] computes a correlation function for the following measure on even-rows Young diagrams

$$
\mathcal{M}(\lambda)=e^{-\eta} \frac{\eta^{n}}{n !} \frac{\operatorname{dim} \lambda}{Z_{n}}
$$

where $Z_{n}$ is the number of standard Young tableaux with $2 n$ entries and even rows. The measure just written above can be understood as the Plancherel measure with the Jack parameter $1 / 2$. The result of Ferrari can be obtained from that of Section 7 of the present paper as follows. Theorem 7.1 gives a correlation kernel for the Plansherel measure defined on the point configurations $\mathcal{D}_{2}(\lambda)=\left\{\lambda_{i}-2 i\right\}$. One can also define a correlation function with point configurations $\tilde{\mathcal{D}}_{2}(\lambda)=\left\{\lambda_{i}-2 i+1, \lambda_{i}-2 i\right\}$, and this correlation function can be determined from the formula in Theorem 7.1. By particle-hole involution this gives a correlation function for point configurations of the form $\left\{-\lambda_{i}^{\prime}+i-1\right\}_{i \geq 1}^{\infty}$. Then the relation $M_{\theta}^{(n)}(\lambda)=M_{\frac{1}{\theta}}^{(n)}\left(\lambda^{\prime}\right)$ enables to obtain the correlation function for the Plancherel measure with the Jack parameter $\theta=1 / 2$.

1.3.6. Special cases of the $z$-measures with $\theta=2,1 / 2$ can be understood as discrete symplectic and orthogonal ensembles. For the explanation of this relation, and for the results on correlation functions of the corresponding discrete ensembles see Borodin and Strahov [14].

Acknowledgment. I am grateful to Alexei Borodin and to Patrik Ferrari for their interest in this work, and for helpful discussions. I also very grateful to reviewers for many valuable comments.

\section{The $z$-MeAsURes With The General PARAMEter $\theta>0$}

We use Macdonald [29] as a basic reference for the notations related to integer partitions and to symmetric functions. In particular, every decomposition

$$
\lambda=\left(\lambda_{1}, \lambda_{2}, \ldots, \lambda_{l}\right): n=\lambda_{1}+\lambda_{2}+\ldots+\lambda_{l},
$$

where $\lambda_{1} \geq \lambda_{2} \geq \ldots \geq \lambda_{l}$ are positive integers, is called an integer partition. We identify integer partitions with the corresponding Young diagrams, and denote the set of all Young diagrams by $\mathbb{Y}$. The set of Young diagrams with $n$ boxes is denoted by $\mathbb{Y}_{n}$. Thus

$$
\mathbb{Y}=\bigcup_{n=0}^{\infty} \mathbb{Y}_{n}
$$


Following Borodin and Olshanski [10], Section 1, let $\mathcal{M}_{z, z^{\prime}, \theta}^{(n)}$ be a complex measure on $\mathbb{Y}_{n}$ defined by

$$
\mathcal{M}_{z, z^{\prime}, \theta}^{(n)}=\frac{n !(z)_{\lambda, \theta}\left(z^{\prime}\right)_{\lambda, \theta}}{(t)_{n} H(\lambda, \theta) H^{\prime}(\lambda, \theta)}
$$

where $n=1,2, \ldots$, and where we use the following notation

- $z, z^{\prime} \in \mathbb{C}$ and $\theta>0$ are parameters, the parameter $t$ is defined by

$$
t=\frac{z z^{\prime}}{\theta}
$$

- $(t)_{n}$ stands for the Pochhammer symbol,

$$
(t)_{n}=t(t+1) \ldots(t+n-1)=\frac{\Gamma(t+n)}{\Gamma(t)} .
$$

- $(z)_{\lambda, \theta}$ is a multidimensional analogue of the Pochhammer symbol defined by

$$
(z)_{\lambda, \theta}=\prod_{(i, j) \in \lambda}(z+(j-1)-(i-1) \theta)=\prod_{i=1}^{l(\lambda)}(z-(i-1) \theta)_{\lambda_{i}} .
$$

Here $(i, j) \in \lambda$ stands for the box in the $i$ th row and the $j$ th column of the Young diagram $\lambda$, and we denote by $l(\lambda)$ the number of nonempty rows in the Young diagram $\lambda$.

$$
\begin{aligned}
& H(\lambda, \theta)=\prod_{(i, j) \in \lambda}\left(\left(\lambda_{i}-j\right)+\left(\lambda_{j}^{\prime}-i\right) \theta+1\right), \\
& H^{\prime}(\lambda, \theta)=\prod_{(i, j) \in \lambda}\left(\left(\lambda_{i}-j\right)+\left(\lambda_{j}^{\prime}-i\right) \theta+\theta\right),
\end{aligned}
$$

where $\lambda^{\prime}$ denotes the transposed diagram.

Proposition 2.1. The following symmetry relations hold true

$$
H(\lambda, \theta)=\theta^{|\lambda|} H^{\prime}\left(\lambda^{\prime}, \frac{1}{\theta}\right), \quad(z)_{\lambda, \theta}=(-\theta)^{|\lambda|}\left(-\frac{z}{\theta}\right)_{\lambda^{\prime}, \frac{1}{\theta}} .
$$

Here $|\lambda|$ stands for the number of boxes in the diagram $\lambda$.

Proof. These relations follow immediately from definitions of $H(\lambda, \theta)$ and $(z)_{\lambda, \theta}$.

Proposition 2.2. We have

$$
\mathcal{M}_{z, z^{\prime}, \theta}^{(n)}(\lambda)=\mathcal{M}_{-z / \theta,-z^{\prime} / \theta, 1 / \theta}^{(n)}\left(\lambda^{\prime}\right) .
$$

Proof. Use definition of $\mathcal{M}_{z, z^{\prime}, \theta}^{(n)}(\lambda)$, equation (2.1), and apply Proposition 2.1. 
Proposition 2.3. We have

$$
\sum_{\lambda \in \mathbb{Y}_{n}} \mathcal{M}_{z, z^{\prime}, \theta}^{(n)}(\lambda)=1
$$

Proof. See Kerov [27], Borodin and Olshanski [10, 11].

Proposition 2.4. Expression (2.1) for $\mathcal{M}_{z, z^{\prime}, \theta}^{(n)}(\lambda)$ is strictly positive for all $n=1,2, \ldots$ and all $\lambda \in \mathbb{Y}_{n}$ if and only if either $z \in \mathbb{C} \backslash\left(\mathbb{Z}_{\leq 0}+\mathbb{Z}_{\geq 0} \theta\right)$ and $z^{\prime}=\bar{z}$, or, under the additional assumption that $\theta$ is rational, both $z, z^{\prime}$ are real numbers lying in one of the intervals between two consecutive numbers from the lattice $\mathbb{Z}+\mathbb{Z} \theta$.

Proof. See Borodin and Olshanski [10].

Clearly, if the conditions in the Proposition above are satisfied, then $\mathcal{M}_{z, z^{\prime}, \theta}^{(n)}$ is a probability measure defined on $\mathbb{Y}_{n}$, as follows from Proposition 2.3 .

It is convenient to mix all measures $\mathcal{M}_{z, z^{\prime}, \theta}^{(n)}$, and to define a new measure $\mathcal{M}_{z, z^{\prime}, \theta, \xi}$ on $\mathbb{Y}=\mathbb{Y}_{0} \cup \mathbb{Y}_{1} \cup \ldots$. Namely, let $\xi \in(0,1)$ be an additional parameter, and set

$$
\mathcal{M}_{z, z^{\prime}, \theta, \xi}(\lambda)=(1-\xi)^{t} \xi^{|\lambda|} \frac{(z)_{\lambda, \theta}\left(z^{\prime}\right)_{\lambda, \theta}}{H(\lambda, \theta) H^{\prime}(\lambda, \theta)} .
$$

We also note the relation

$$
\mathcal{M}_{z, z^{\prime}, \theta, \xi}(\lambda)=(1-\xi)^{t} \xi^{n} \frac{(t)_{n}}{n !} \mathcal{M}_{z, z^{\prime}, \theta, \xi}^{(n)}(\lambda), \quad|\lambda|=n
$$

Proposition 2.5. We have

$$
\sum_{\lambda \in \mathbb{Y}} \mathcal{M}_{z, z^{\prime}, \theta, \xi}(\lambda)=1
$$

Proof. Follows immediately from Proposition 2.3.

If conditions on $z, z^{\prime}$ formulated in Propositions 1.2, 1.3 in Borodin and Olshanski [10] are satisfied, then $\mathcal{M}_{z, z^{\prime}, \theta, \xi}(\lambda)$ is a probability measure on $\mathbb{Y}$. We will refer to $\mathcal{M}_{z, z^{\prime}, \theta, \xi}(\lambda)$ as to the $z$-measure with the deformation (Jack) parameter $\theta$.

When both $z, z^{\prime}$ go to infinity, expression (2.1) has a limit

$$
\mathcal{M}_{\infty, \infty, \theta}^{(n)}(\lambda)=\frac{n ! \theta^{n}}{H(\lambda, \theta) H^{\prime}(\lambda, \theta)}
$$

called the Plancherel measure on $\mathbb{Y}_{n}$ with general $\theta>0$. Instead of (2.4), sometimes it is more convenient to consider the Poissonized 
Plancherel measure with general $\theta>0$,

$$
\mathcal{M}_{\infty, \infty, \theta, \eta}(\lambda)=e^{-\eta^{2}}\left(\eta^{2}\right)^{|\lambda|} \frac{\theta^{|\lambda|}}{H(\lambda, \theta) H^{\prime}(\lambda, \theta)},
$$

where $\eta$ is a real parameter.

\section{MAin RESUlT}

Set $\mathcal{D}_{2}(\lambda)=\left\{\lambda_{i}-2 i\right\}$. Thus $\mathcal{D}_{2}(\lambda)$ is an infinite subset of $\mathbb{Z}$ corresponding to the Young diagram $\lambda$ containing infinitely many negative integers. Let $X=\left(x_{1}, \ldots, x_{n}\right)$ be a subset of $\mathbb{Z}$ consisting of $n$ pairwise distinct points, and define

$$
\varrho_{z, z^{\prime}, 2, \xi}(X)=\mathcal{M}_{z, z^{\prime}, 2, \xi}\left(\left\{\lambda \mid X \subset \mathcal{D}_{2}(\lambda)\right\}\right) .
$$

If $\mathcal{M}_{z, z^{\prime}, \theta, \xi}$ is positive definite, then it is a probability measure defined on $\mathbb{Y}$, and $\varrho_{z, z^{\prime}, \theta, \xi}(X)$ is the probability that the random point configuration $\mathcal{D}_{\theta}(\lambda)$ contains the fixed $n$-point configuration $X=\left(x_{1}, \ldots, x_{n}\right)$. Our goal here is to prove the following

Theorem 3.1. a) We have

$$
\varrho_{z, z-1,2, \xi}(X)=\operatorname{Pf}\left[K_{z, z-1,2, \xi}\left(x_{i}, x_{j}\right)\right]_{i, j=1}^{n},
$$

where the $2 \times 2$ matrix valued kernel, $K_{z, z-1,2, \xi}(x, y)$, can be written as

$$
K_{z, z-1,2, \xi}(x, y)=\left[\begin{array}{cc}
S_{z, z-1,2, \xi}(x+1, y+1) & -S_{z, z-1,2, \xi}(x+1, y) \\
-S_{z, z-1,2, \xi}(x, y+1) & S_{z, z-1,2, \xi}(x, y)
\end{array}\right] .
$$

The function $S_{z, z-1,2, \xi}(x, y)$ has the following contour integral representation

$$
\begin{aligned}
S_{z, z-1,2, \xi}(x, y)=\frac{1}{(2 \pi i)^{2}} \oint_{\left\{w_{1}\right\}} \oint_{\left\{w_{2}\right\}} \frac{\left(1+\sqrt{\xi} w_{1}\right)^{-z}\left(1+\sqrt{\xi} w_{2}\right)^{-z}\left(1+\frac{\sqrt{\xi}}{w_{1}}\right)^{z}\left(1+\frac{\sqrt{\xi}}{w_{2}}\right)^{z}}{\left(w_{2} w_{1}-1\right)} \\
\times \frac{\left(w_{2}-w_{1}\right)}{\left(w_{2}^{2}-1\right)\left(w_{1}^{2}-1\right)} \frac{d w_{1} d w_{2}}{w_{1}^{x} w_{2}^{y}},
\end{aligned}
$$

where $\left\{w_{1}\right\},\left\{w_{2}\right\}$ are arbitrary simple contours satisfying the conditions

- both contours go around 0 in positive direction;

- the unit circle is contained in the interior of the contour $\left\{w_{1}\right\}$, and in the interior of the contour $\left\{w_{2}\right\}$, so $\left|w_{1}\right|>1,\left|w_{2}\right|>1$.

- the point $\xi^{-1 / 2}$ lies outside both contours $\left\{w_{1}\right\}$ and $\left\{w_{2}\right\}$. 
b) The function $S_{z, z-1,2, \xi}(x, y)$ also can be written as

$$
\begin{aligned}
& S_{z, z-1,2, \xi}(x, y)=(1-\xi)^{2 z} \sum_{k, m=1}^{\infty}(-1)^{k+m+x+y} \xi^{\frac{k+m+x+y}{2}}(\Upsilon)_{k, m}(z)_{k+x}(z)_{m+y} \\
& \frac{F\left(-z+1,-z ; k+x+1 ; \frac{\xi}{\xi-1}\right)}{\Gamma(k+x+1)} \frac{F\left(-z+1,-z ; m+y+1 ; \frac{\xi}{\xi-1}\right)}{\Gamma(m+y+1)}
\end{aligned}
$$

where $F(a, b ; c ; w)$ denotes the Gauss hypergeometric function, and $(\Upsilon)_{k, m}$ are the matrix elements of the matrix $\Upsilon$ defined by

$$
\Upsilon=\left[\begin{array}{ccccccccc}
0 & -1 & 0 & -1 & 0 & -1 & 0 & -1 & \cdots \\
1 & 0 & 0 & 0 & 0 & 0 & 0 & 0 & \cdots \\
0 & 0 & 0 & -1 & 0 & -1 & 0 & -1 & \cdots \\
1 & 0 & 1 & 0 & 0 & 0 & 0 & 0 & \cdots \\
0 & 0 & 0 & 0 & 0 & -1 & 0 & -1 & \cdots \\
1 & 0 & 1 & 0 & 1 & 0 & 0 & 0 & \ldots \\
0 & 0 & 0 & 0 & 0 & 0 & 0 & -1 & \ldots \\
1 & 0 & 1 & 0 & 1 & 0 & 1 & 0 & \cdots \\
\vdots & \vdots & \vdots & \vdots & \vdots & \vdots & \vdots & \vdots & \ddots
\end{array}\right] .
$$

That is, $\Upsilon$ is an antisymmetric matrix whose entries are defined by the relations

$$
\begin{gathered}
\Upsilon(2 i+1,2 j+1)=\Upsilon(2 i, 2 j)=0, \text { for any } i, j \geq 0 \\
\Upsilon(2 i+1,2 j+2)=0, \text { for } 0 \leq i \leq j \\
\Upsilon(2 i, 2 j+1)=-1, \text { for } 0 \leq i \leq j
\end{gathered}
$$

Remark 3.2. 1) Since $\xi \in(0,1)$, we have $\xi /(\xi-1)<0$, and the function $w \rightarrow F(a, b ; c ; w)$ is well defined on the negative semi-axis $w<0$. The function $F(a, b ; c ; w)$ is not defined at $c=0,-1,-2, \ldots$, but the ratio $F(a, b ; c ; w) / \Gamma(c)$ is well defined for all $c \in \mathbb{C}$.

2) The matrix $\Upsilon$ appears in the study of the random matrix ensembles of $\beta=1,4$ symmetry classes, and of their discrete analogues, see Borodin and Strahov [13], Section 6, and also Borodin and Strahov [14], the proof of Lemma 6.1.

\section{Pfaffian Schur measures}

In the case when $z^{\prime}=z-1$ the measure $\mathcal{M}_{z, z-1,2, \xi}$ can be written as a determinant, see Proposition 6.1. This will enable us to obtain Theorem 3.1 as a corollary of a more general algebraic fact.

Let $\Lambda$ denote the algebra of symmetric functions. The algebra $\Lambda$ can be considered as the algebra of polynomials $\mathbb{C}\left[p_{1}, p_{2}, \ldots\right]$ in power sums $p_{1}, p_{2}, \ldots$ Then it can be realized, in different ways, as an algebra 
of functions, depending on a specialization of the generators $p_{k}$. The elements $h_{k}$ and $e_{k}$ (the complete homogeneous symmetric functions and the elementary symmetric functions) can be introduced through the generating series:

$$
1+\sum_{k=1}^{\infty} h_{k} u^{k}=\exp \left(\sum_{k=1}^{\infty} p_{k} \frac{u^{k}}{k}\right)=\left(1+\sum_{k=1}^{\infty} e_{k}(-u)^{k}\right)^{-1} .
$$

We define the generating series for $\left\{h_{k}\right\}$ and $\left\{e_{k}\right\}$ as formal series in a complex variable $u$ by

$$
H(u)=1+\sum_{k=1}^{\infty} h_{k} u^{k}, \quad E(u)=1+\sum_{k=1}^{\infty} e_{k} u^{k} .
$$

The Schur function $s_{\lambda}$ indexed by a Young diagram $\lambda$ can be introduced through the Jacobi-Trudi formula:

$$
s_{\lambda}=\operatorname{det}\left[h_{\lambda_{i}-i+j}\right] \text {, }
$$

where, by convention, $h_{0}=1, h_{-1}=h_{-2}=\ldots=0$, and the order of the determinant is any number greater or equal to $l(\lambda)$.

By a specialization $\pi$ of the algebra of symmetric function $\Lambda$ we mean a homomorphism to $\mathbb{C}$.

Let $\pi$ be an arbitrary specialization of the algebra $\Lambda$ of symmetric functions. Introduce the complex measure

$$
\mathcal{M}_{\text {Schur }, 2}(\lambda)=\frac{1}{\mathcal{Z}_{\text {Schur }, 2}} \operatorname{det}\left[\pi\left\{e_{\lambda_{j}-2 j+i+1}\right\}, \pi\left\{e_{\lambda_{j}-2 j+i}\right\}\right]
$$

where $1 \leq j \leq l(\lambda), 1 \leq i \leq 2 l(\lambda)$, and

$$
\mathcal{Z}_{\text {Schur }, 2}=\sum_{\lambda \in \mathbb{Y}} \operatorname{det}\left[\pi\left\{e_{\lambda_{j}-2 j+i+1}\right\}, \pi\left\{e_{\lambda_{j}-2 j+i}\right\}\right]
$$

is assumed to be absolutely convergent. Now we prove the following

Theorem 4.1. a) For any measure $\mathcal{M}_{\text {Schur,2 }}$ on the set $\mathbb{Y}$ of all Young diagrams, and for any fixed subset $X=\left\{x_{1}, \ldots, x_{n}\right\}$ of $\mathbb{Z}$ containing pairwise distinct points we have the following formal series identity

$$
\begin{aligned}
\sum_{X \subset \mathcal{D}_{2}(\lambda)} \operatorname{det} & {\left[\pi\left\{e_{\lambda_{j}-2 j+i+1}\right\}, \pi\left\{e_{\lambda_{j}-2 j+i}\right\}\right] } \\
= & \mathcal{Z}_{\text {Schur }, 2} \operatorname{Pf}\left[K_{\text {Schur }, 2}\left(x_{i}, x_{j}\right)\right]_{i, j=1}^{n} .
\end{aligned}
$$

Here the kernel $K_{\text {Schur, } 2}(x, y)$ can be written as

$$
K_{\text {Schur }, 2}(x, y)=\left[\begin{array}{cc}
S_{\text {Schur }, 2}(x+1, y+1) & -S_{\text {Schur }, 2}(x+1, y) \\
-S_{\text {Schur }, 2}(x, y+1) & S_{\text {Schur }, 2}(x, y)
\end{array}\right],
$$


and the function $S_{\mathrm{Schur}, 2}(x, y)$ admits the following integral representation

$$
\begin{aligned}
& S_{\text {Schur }, 2}(x, y) \\
& =\frac{1}{(2 \pi i)^{2}} \oint_{\left\{w_{1}\right\}} \oint_{\left\{w_{2}\right\}} \frac{d w_{1} d w_{2}}{w_{1}^{x} w_{2}^{y}} \frac{\pi\left\{E\left(w_{1}\right)\right\}}{\pi\left\{E\left(w_{1}^{-1}\right)\right\}} \frac{\pi\left\{E\left(w_{2}\right)\right\}}{\pi\left\{E\left(w_{2}^{-1}\right)\right\}} \frac{\left(w_{2}-w_{1}\right)}{\left(w_{2} w_{1}-1\right)\left(w_{2}^{2}-1\right)\left(w_{1}^{2}-1\right)},
\end{aligned}
$$

where $\pi\{E(w)\}=1+\sum_{k=1}^{\infty} \pi\left\{e_{k}\right\} w^{k}$, and where $\left\{w_{1}\right\},\left\{w_{2}\right\}$ are simple contours which both go around 0 in positive direction, they are chosen in such a way that they do not include the possible poles at $\pi\left\{E\left(w_{1}^{-1}\right)\right\}, \pi\left\{E\left(w_{2}^{-1}\right)\right\}$, and $\left|w_{1}\right|>1,\left|w_{2}\right|>1$.

b) The function $S_{\mathrm{Schur}, 2}(x, y)$ also admits the representation

$$
S_{\text {Schur }, 2}(x, y)=\sum_{k, m=1}^{\infty} \Phi_{k}(x)(\Upsilon)_{k, m} \Phi_{m}(y),
$$

where the functions $\Phi_{k}$ are given by

$$
\Phi_{k}(x)=\frac{1}{2 \pi i} \oint_{\{w\}} \frac{\pi\{E(w)\}}{\pi\left\{E\left(\frac{1}{w}\right)\right\}} \frac{d w}{w^{k+x+1}} .
$$

In the formulae just written above $\{w\}$ is a simple contour which goes around 0 in positive direction, and $(\Upsilon)_{k, m}$ are the matrix elements of the matrix $\Upsilon$ defined by equation (3.1).

Remark 4.2. For a specific specialization $\pi$ of $\Lambda$ one can investigate conditions under which equations (4.2), (4.3) become numerical equalities.

Define the correlation function of $\mathcal{M}_{\text {Schur,2 }}$ by

$$
\varrho_{\text {Schur }, 2}(X)=\mathcal{M}_{\text {Schur }, 2}\left(\left\{\lambda \mid X \subset \mathcal{D}_{2}(\lambda)\right\}\right)
$$

If $\mathcal{M}_{\text {Schur,2 }}$ is positive definite, then it is a probability measure defined on $\mathbb{Y}$, and $\varrho_{\text {Schur }, 2}(X)$ is the probability that the random point configuration $\mathcal{D}_{2}(\lambda)$ contains the fixed $n$-point configuration $X=\left(x_{1}, \ldots, x_{n}\right)$. By Theorem 4.1 we have

$$
\varrho_{\text {Schur }, 2}(X)=\operatorname{Pf}\left[K_{\text {Schur }, 2}\left(x_{i}, x_{j}\right)\right]_{i, j=1}^{n},
$$

where the correlation kernel, $K_{\text {Schur,2 }}(x, y)$, is given by formulae (4.2), (4.3). 


\section{Proof of Theorem 4.1}

Let $X=\left(x_{1}, \ldots, x_{n}\right)$ be a fixed subset of $\mathbb{Z}$ consisting of $n$ pairwise distinct points. Let us pick an integer $N$ such that $N>n$, and such that $X$ is a subset of $\{-2 N,-2 N+1, \ldots\}$. Define

$$
\varrho_{\text {Schur }, 2}^{(N)}(X)=\frac{\mathcal{M}_{\text {Schur }, 2}\left\{\lambda \mid \lambda \in \mathbb{Y}(N), X \subset\left\{\lambda_{i}-2 i\right\}\right\}}{\mathcal{M}_{\text {Schur }, 2}\{\lambda \mid \lambda \in \mathbb{Y}(N)\}} .
$$

Here $\mathbb{Y}(N)$ denotes the subset of $\mathbb{Y}$ consisting of Young diagrams whith number of rows less or equal to $N$. Clearly, $\varrho_{\text {Schur, } 2}^{(N)}(X)$ converges to $\varrho_{\text {Schur }, 2}(X)$ in the statement of Theorem 4.1 as $N \longrightarrow \infty$.

For a given Young diagram $\lambda$ from $\mathbb{Y}(N)$ we define $l_{j}=\lambda_{j}-2 j$, $1 \leq j \leq N$ (if the length $l(\lambda)$ of the diagram $\lambda$ is less then $N$ we set $\left.\lambda_{l(\lambda)+1}=0, \lambda_{l(\lambda)+2}=0, \ldots, \lambda_{N}=0\right)$. We also introduce functions $\phi_{i}(l)$ and $\psi_{i}(l)$ on $\mathbb{Z}$ (where $1 \leq i \leq 2 l(\lambda)$ ) by formulae $\phi_{i}(l)=\pi\left\{e_{l+i+1}\right\}$, $\psi_{i}(l)=\pi\left\{e_{l+i}\right\}$, where $\pi$ is a specialization of the algebra $\Lambda$ of symmetric functions. With this notation we can rewrite $\varrho_{\text {Schur,2 }}^{(N)}(X)$ as follows

$$
\varrho_{\text {Schur }, 2}^{(N)}(X)=\frac{\sum_{\substack{l_{1}>\ldots>l_{N} \geq-2 N \\ X \subset\left\{l_{1}, \ldots, l_{N}\right\}}} \operatorname{det}\left[\phi_{i}\left(l_{j}\right), \psi_{i}\left(l_{j}\right)\right]}{\sum_{l_{1}>\ldots>l_{N} \geq-2 N} \operatorname{det}\left[\phi_{i}\left(l_{j}\right), \psi_{i}\left(l_{j}\right)\right]},
$$

where $1 \leq i \leq 2 N, 1 \leq j \leq N$. By methods of Random Matrix Theory (see, for example, Tracy and Widom [42], Section 8) we can represent $\varrho_{\text {Schur }, 2}^{(N)}(X)$ as a Pfaffian of a $2 \times 2$ matrix valued kernel. Namely, we can obtain the following Lemma

Lemma 5.1. Suppose that $X$ and $N$ are chosen as described above, and suppose that $\varrho^{(N)}(X)$ is defined by equation (5.1), where $\phi_{i}(l), \psi_{i}(l)$ are now arbitrary functions of finite support defined on $\mathbb{Z}$, and $1 \leq i \leq N$. The following identity holds true

$$
\varrho^{(N)}(X)=\operatorname{Pf}\left[K^{(N)}\left(x_{i}, x_{j}\right)\right]_{i, j=1}^{n},
$$

where $K^{(N)}(x, y)$ is a $2 \times 2$ matrix valued kernel defined by the formula

$$
\begin{aligned}
& K^{(N)}(x, y)=\left[\begin{array}{cc}
\sum_{i, j=1}^{2 N} \phi_{i}(x)\left((M(N))^{-1}\right)_{i, j} \phi_{j}(y) & -\sum_{i, j=1}^{2 N} \phi_{i}(x)\left((M(N))^{-1}\right)_{i, j} \psi_{j}(y) \\
-\sum_{i, j=1}^{2 N} \psi_{i}(x)\left((M(N))^{-1}\right)_{i, j} \phi_{j}(y) & \sum_{i, j=1}^{2 M} \psi_{i}(x)\left((M(N))^{-1}\right)_{i, j} \psi_{j}(y)
\end{array}\right], \\
& \text { where }(M(N))_{i, j}=\sum_{x=-2 N}^{+\infty}\left(\phi_{i}(x) \psi_{j}(x)-\phi_{j}(x) \psi_{i}(x)\right), 1 \leq i, j \leq 2 N .
\end{aligned}
$$


In our case, Lemma 5.1 (with $\phi_{i}(x)=\pi\left(e_{x+i+1}\right)$ and $\psi_{i}(x)=\pi\left(e_{x+i}\right)$, where $x \in \mathbb{Z})$ gives the following representation for the correlation function $\varrho_{\mathrm{Schur}, 2}^{(N)}(X)$

$$
\varrho_{\text {Schur }, 2}^{(N)}(X)=\operatorname{Pf}\left[K_{\text {Schur }, 2}^{(N)}\left(x_{i}, x_{j}\right)\right]_{i, j=1}^{n},
$$

where

$$
K_{\mathrm{Schur}, 2}^{(N)}(x, y)=\left[\begin{array}{cc}
S_{\mathrm{Schur}, 2}^{(N)}(x+1, y+1) & -S_{\mathrm{Schur}, 2}^{(N)}(x+1, y) \\
-S_{\mathrm{Schur}, 2}^{(N)}(x, y+1) & S_{\mathrm{Schur}, 2}^{(N)}(x, y)
\end{array}\right],
$$

and where the function $S_{\mathrm{Schur}, 2}^{(N)}(x, y)$ is defined by

$$
S_{\text {Schur }, 2}^{(N)}(x, y)=\sum_{i, j=1}^{2 N} \pi\left(e_{x+i+1}\right)\left((M(N))^{-1}\right)_{i, j} \pi\left(e_{y+j+1}\right) .
$$

In the formulae above the matrix $M(N)$ is a $2 N \times 2 N$ matrix whose matrix coefficients are defined by the formula

$$
(M(N))_{i, j}=\sum_{x=-2 N}^{+\infty}\left(\pi\left(e_{x+i+1}\right) \pi\left(e_{x+j}\right)-\pi\left(e_{x+j+1}\right) \pi\left(e_{x+i}\right)\right), 1 \leq i, j \leq 2 N .
$$

As $\pi\left\{e_{-k}\right\}=0$ for $k=1,2, \ldots$ we can rewrite the formula for the matrix coefficients $(M(N))_{i, j}$ as follows

$$
(M(N))_{i, j}=\sum_{x=-\infty}^{+\infty}\left(\pi\left(e_{x+i+1}\right) \pi\left(e_{x+j}\right)-\pi\left(e_{x+i}\right) \pi\left(e_{x+j+1}\right)\right), 1 \leq i, j \leq 2 N .
$$

Clearly, the matrix $M(N)$ can be understood as the $N$ th principal minor of the infinite matrix $M^{\infty}$ whose matrix elements are defined by the formula

$$
\left(M^{\infty}\right)_{i, j}=\sum_{x=-\infty}^{+\infty}\left(\pi\left(e_{x+i+1}\right) \pi\left(e_{x+j}\right)-\pi\left(e_{x+i}\right) \pi\left(e_{x+j+1}\right)\right), 1 \leq i, j .
$$

Rains [39] has shown that for matrices of this type

$$
\lim _{N \rightarrow \infty}\left((M(N))^{-1}-\left(M^{\infty}\right)^{-1}\right)_{i, j}=0
$$

for any fixed $i, j$, see Lemma 2.1, equations (3.12), (4.23) in Rains [39]. We then deduce that the kernel $K_{\text {Schur,2 }}(x, y)$ can be written as

$$
K_{\text {Schur }, 2}(x, y)=\left[\begin{array}{cc}
S_{\text {Schur }, 2}(x, y) & -S_{\text {Schur }, 2}(x+1, y) \\
-S_{\text {Schur }, 2}(x, y) & S_{\text {Schur }, 2}(x, y)
\end{array}\right],
$$


where

$$
S_{\text {Schur }, 2}(x, y)=\sum_{i, j=1}^{\infty} \pi\left(e_{x+i}\right)\left(\left(M^{\infty}\right)^{-1}\right)_{i, j} \pi\left(e_{y+j}\right),
$$

and the semi-infinite matrix $M^{\infty}$ is defined by equation (5.3).

It is convenient to rewrite formula (5.3). Set $k=x+i+j$. Note that $k$ can be any (negative or positive) integer. We have $x+i=k-j$, and $x+j=k-i$. Then $\left(M^{\infty}\right)_{i, j}$ can be rewritten as

$$
\left(M^{\infty}\right)_{i, j}=\sum_{k=-\infty}^{+\infty}\left(\pi\left(e_{k-j+1}\right) \pi\left(e_{k-i}\right)-\pi\left(e_{k-j}\right) \pi\left(e_{k-i+1}\right)\right), 1 \leq i, j .
$$

As soon as $\pi\left\{e_{-k}\right\}=0$, for $k=1,2, \ldots$, we can also represent $\left(M^{\infty}\right)_{i, j}$ as

$$
\left(M^{\infty}\right)_{i, j}=\sum_{k=1}^{+\infty}\left(\pi\left(e_{k-j+1}\right) \pi\left(e_{k-i}\right)-\pi\left(e_{k-j}\right) \pi\left(e_{k-i+1}\right)\right), 1 \leq i, j .
$$

Let $\varphi(z)$ be a formal series of the form

$$
\varphi(z)=\sum_{n \in \mathbb{Z}} \varphi_{n} z^{n}
$$

It is convenient to introduce the following notation. Let $\left[z^{n}\right] \varphi$ stand for the coefficient of $z^{n}$ in $\varphi$, and let $T \varphi$ denote the Toeplitz semi-infinite matrix defined by $\varphi$. The matrix elements of $T \varphi$ are

$$
(T \varphi)_{i, j}=\varphi_{j-i}=\left[z^{j-i}\right] \varphi=\frac{1}{2 \pi i} \oint_{\{w\}} w^{j-i} \varphi(w) \frac{d w}{w}
$$

where $\{w\}$ is an arbitrary simple contour which goes around 0 in positive direction.

With the above notation rewrite the expression for the matrix elements $\left(M^{\infty}\right)_{i, j}$ as follows

$$
\left(M^{\infty}\right)_{i, j}=\sum_{k=1}^{+\infty}(T \pi\{E(z)\})_{j, k+1}(T \pi\{E(z)\})_{i, k}-(T \pi\{E(z)\})_{i, k+1}(T \pi\{E(z)\})_{j, k}
$$

where $1 \leq i, j$.

Up to now we have used essentially the same arguments as in Rains [39]. In what follows we show that $S_{\text {Schur }, 2}(x, y)$ (equation (5.4)) admits the contour integral representation as in Theorem 4.1.

Introduce a semi-infinite matrix $D$ defining its matrix elements by

$$
(D)_{k, m}=\delta_{k+1, m}-\delta_{k, m+1}, \quad 1 \leq k, m .
$$


Then we obtain

$$
\left(M^{\infty}\right)_{i, j}=\sum_{k, m=1}^{+\infty}(T \pi\{E(z)\})_{i, k}(D)_{k, m}(T \pi\{E(z)\})_{j, m}, 1 \leq i, j .
$$

Therefore, we have $M^{\infty}=T \pi\{E(z)\} D(T \pi\{E(z)\})^{t}$. Note that $D$ has the following form

$$
D=\left[\begin{array}{ccccccccc}
0 & 1 & 0 & 0 & 0 & 0 & 0 & 0 & \cdots \\
-1 & 0 & 1 & 0 & 0 & 0 & 0 & 0 & \cdots \\
0 & -1 & 0 & 1 & 0 & 0 & 0 & 0 & \cdots \\
0 & 0 & -1 & 0 & 1 & 0 & 0 & 0 & \cdots \\
0 & 0 & 0 & -1 & 0 & 1 & 0 & 0 & \cdots \\
0 & 0 & 0 & 0 & -1 & 0 & 1 & 0 & \cdots \\
0 & 0 & 0 & 0 & 0 & -1 & 0 & 1 & \cdots \\
0 & 0 & 0 & 0 & 0 & 0 & -1 & 0 & \cdots \\
\vdots & \vdots & \vdots & \vdots & \vdots & \ddots & \ddots & \ddots & \ddots
\end{array}\right]
$$

This matrix has inverse, which is denoted by $\Upsilon$, whose explicit form is given by equation (3.1).

Now we can write $\left(M^{\infty}\right)^{-1}=\left[(T \pi\{E(z)\})^{t}\right]^{-1} \Upsilon[T \pi\{E(z)\}]^{-1}$, and we obtain the following expression for the matrix elements of $\left(M^{\infty}\right)^{-1}$

$$
\begin{aligned}
& \left(\left(M^{\infty}\right)^{-1}\right)_{i, j} \\
& =\sum_{k, m=1}^{\infty}\left(\left[(T \pi\{E(z)\})^{t}\right]^{-1}\right)_{i, k}(\Upsilon)_{k, m}\left([T \pi\{E(z)\}]^{-1}\right)_{m, j},
\end{aligned}
$$

where $i, j \geq 1$.

Lemma 5.2. For any integers $j, m$ such that $j, m \geq 1$

$$
\left([T \pi\{E(z)\}]^{-1}\right)_{j, m}=\left[z^{m-j}\right]\left(\frac{1}{\pi\{E(z)\}}\right) .
$$

Proof. We need to check that

$$
\sum_{k=1}^{\infty}\left([T \pi\{E(z)\}]^{-1}\right)_{j, k}([T \pi\{E(z)\}])_{k, m}=\delta_{j, m}
$$

remains to be valid, if we replace $\left([T \pi\{E(z)\}]^{-1}\right)_{j, k}$ by $\left[z^{k-j}\right]\left(\frac{1}{\pi\{E(z)\}}\right)$.

Note that

$$
\left[z^{k}\right]\left(\frac{1}{\pi\{E(z)\}}\right)=0, \text { for } k \leq-1
$$

and

$$
\left[z^{k}\right](\pi\{E(z)\})=0, \text { for } k \leq-1
$$


Now the left-hand side of equation (5.7) can be written as

$$
\begin{aligned}
& \sum_{k=1}^{\infty}\left[z^{k-j}\right]\left(\frac{1}{\pi\{E(z)\}}\right)([T \pi\{E(z)\}])_{k, m}=\sum_{k=1}^{\infty}\left[z^{k-j}\right]\left(\frac{1}{\pi\{E(z)\}}\right)\left[z^{m-k}\right](\pi\{E(z)\}) \\
& =\sum_{k=j}^{\infty}\left[z^{k-j}\right]\left(\frac{1}{\pi\{E(z)\}}\right)\left[z^{m-k}\right](\pi\{E(z)\})=\sum_{n=0}^{\infty}\left[z^{n}\right]\left(\frac{1}{\pi\{E(z)\}}\right)\left[z^{m-n-j}\right](\pi\{E(z)\}) .
\end{aligned}
$$

Taking into account the formula

$$
\left[z^{k}\right](A(z) B(z))=\sum_{n=0}^{\infty}\left[z^{n}\right] A(z)\left[z^{k-n}\right] B(z),
$$

we obtain that $\sum_{k=1}^{\infty}\left[z^{k-j}\right]\left(\frac{1}{\pi\{E(z)\}}\right)([T \pi\{E(z)\}])_{k, m}$ equals $\delta_{j, m}$.

Now we are ready to derive the contour integral representation for the kernel $S_{\text {Schur, } 2}(x, y)$. Equations (5.5), (5.6) imply formula (4.4), with $\Phi_{k}$ defined by the formula

$$
\Phi_{k}(x)=\sum_{i=1}^{\infty}\left[z^{x+i}\right] \pi\{E(z)\}\left[z^{i-k}\right]\left(\frac{1}{\pi\{E(z)\}}\right), k \geq 1 .
$$

It is possible to represent $\Phi_{k}(x)$ as a contour integral, see equation (4.5). To see this rewrite formula (5.8) as follows

$$
\Phi_{k}(x)=\sum_{i=-\infty}^{\infty}\left[z^{x+i}\right] \pi\{E(z)\}\left[z^{i-k}\right]\left(\frac{1}{\pi\{E(z)\}}\right),
$$

where we have used the fact that the formal series $(\pi\{E(z)\})^{-1}$ does not contain terms of the form $a_{n} z^{-n}, n=1,2, \ldots$. Changing the index of the summation, and applying formulae

$$
\left[z^{k}\right] A\left(z^{-1}\right)=\left[z^{-k}\right] A(z),
$$

and

$$
\left[z^{k}\right](A(z) B(z))=\sum_{i=-\infty}^{\infty}\left[z^{i}\right] A(z)\left[z^{k-i}\right] B(z)
$$

we find

$$
\Phi_{k}(x)=\left[z^{x+k}\right]\left[\frac{\pi\{E(z)\}}{\pi\left\{E\left(z^{-1}\right)\right\}}\right] .
$$


This formula is equivalent to equation (4.5). Using (4.5) we can represent $S_{\text {Schur,2 }}(x, y)$ as follows

$S_{\text {Schur }, 2}(x, y)=\frac{1}{(2 \pi i)^{2}} \oint_{\left\{w_{1}\right\}} \oint_{\left\{w_{2}\right\}} \frac{\pi\left\{E\left(w_{1}\right)\right\}}{\pi\left\{E\left(\frac{1}{w_{1}}\right)\right\}} \frac{\pi\left\{E\left(w_{2}\right)\right\}}{\pi\left\{E\left(\frac{1}{w_{2}}\right)\right\}}\left(\sum_{k, m=1}^{\infty} \frac{(\Upsilon)_{k, m}}{w_{1}^{k} w_{2}^{m}}\right) \frac{d w_{1}}{w_{1}^{x+1}} \frac{d w_{2}}{w_{2}^{y+1}}$

where $\left\{w_{1}\right\},\left\{w_{2}\right\}$ are simple contours which go around 0 in positive direction.

The proof of Theorem 4.1 is accomplished by the following

Proposition 5.3. Assume that $\left|w_{1}\right|>1,\left|w_{2}\right|>1$, and let $\Upsilon$ be a semi-infinite matrix defined by equation (3.1). Then

$$
\sum_{k, m=1}^{\infty} \frac{(\Upsilon)_{k, m}}{w_{1}^{k} w_{2}^{m}}=\frac{w_{2} w_{1}\left(w_{2}-w_{1}\right)}{\left(w_{2} w_{1}-1\right)\left(w_{2}^{2}-1\right)\left(w_{1}^{2}-1\right)}
$$

Proof. It is convenient to represent the sum in the left-hand side of the equation just written above as

$$
\left[\begin{array}{llll}
\frac{1}{w_{1}} & \frac{1}{w_{1}^{2}} & \frac{1}{w_{1}^{3}} & \cdots
\end{array}\right] \Upsilon\left[\begin{array}{c}
\frac{1}{w_{2}} \\
\frac{1}{w_{2}^{2}} \\
\frac{1}{w_{2}^{3}} \\
\vdots
\end{array}\right] .
$$


Using the explicit form of $\Upsilon$ (see equation (3.1)) we find that this matrix product equals

$$
\begin{aligned}
& \frac{1}{w_{1}}\left(-\frac{1}{w_{2}^{2}}-\frac{1}{w_{2}^{4}}-\frac{1}{w_{2}^{6}}-\frac{1}{w_{2}^{8}}-\ldots\right) \\
& +\frac{1}{w_{1}^{2}} \frac{1}{w_{2}} \\
& +\frac{1}{w_{1}^{3}}\left(-\frac{1}{w_{2}^{4}}-\frac{1}{w_{2}^{6}}-\frac{1}{w_{2}^{8}}-\frac{1}{w_{2}^{10}}-\ldots\right) \\
& +\frac{1}{w_{1}^{4}} \frac{1}{w_{2}}+\frac{1}{w_{1}^{4}} \frac{1}{w_{2}^{3}} \\
& +\frac{1}{w_{1}^{5}}\left(-\frac{1}{w_{2}^{6}}-\frac{1}{w_{2}^{8}}-\frac{1}{w_{2}^{10}}-\frac{1}{w_{2}^{12}}-\ldots\right) \\
& +\frac{1}{w_{1}^{6}} \frac{1}{w_{2}}+\frac{1}{w_{1}^{6}} \frac{1}{w_{2}^{3}}+\frac{1}{w_{1}^{6}} \frac{1}{w_{2}^{5}} \\
& +\frac{1}{w_{1}^{7}}\left(-\frac{1}{w_{2}^{8}}-\frac{1}{w_{2}^{10}}-\frac{1}{w_{2}^{12}}-\frac{1}{w_{2}^{14}}-\ldots\right) \\
& +\frac{1}{w_{1}^{8}} \frac{1}{w_{2}}+\frac{1}{w_{1}^{8}} \frac{1}{w_{2}^{3}}+\frac{1}{w_{1}^{8}} \frac{1}{w_{2}^{5}}+\frac{1}{w_{1}^{8}} \frac{1}{w_{2}^{7}} \\
& +\ldots \\
& =-\frac{1}{w_{1} w_{2}^{2}} \frac{1}{1-\frac{1}{w_{2}^{2}}}-\frac{1}{w_{1}^{3} w_{2}^{4}} \frac{1}{1-\frac{1}{w_{2}^{2}}}-\frac{1}{w_{1}^{5} w_{2}^{6}} \frac{1}{1-\frac{1}{w_{2}^{2}}}-\ldots \\
& +\frac{1}{w_{1}^{2} w_{2}} \frac{1}{1-\frac{1}{w_{1}^{2}}}+\frac{1}{w_{1}^{4} w_{2}^{3}} \frac{1}{1-\frac{1}{w_{1}^{2}}}+\frac{1}{w_{1}^{6} w_{2}^{5}} \frac{1}{1-\frac{1}{w_{1}^{2}}}+\ldots \\
& =
\end{aligned}
$$

\section{Proof of Theorem 3.1}

Proposition 6.1. We have

$$
\begin{aligned}
\mathcal{M}_{z, z-1,2, \xi}(\lambda)= & (1-\xi)^{\frac{z(z-1)}{2}} \xi^{|\lambda|}(z)_{\lambda, 2}(z-1)_{\lambda, 2} \\
& \times \operatorname{det}\left[\frac{1}{\left(\lambda_{j}-2 j+i+1\right) !}, \frac{1}{\left(\lambda_{j}-2 j+i\right) !}\right],
\end{aligned}
$$

where $1 \leq i \leq 2 l(\lambda)$, and $1 \leq j \leq l(\lambda)$.

Proof. Using the explicit formula for $H(\lambda, 2) H^{\prime}(\lambda, 2)$ given in the proof

of Lemma 3.5 in Borodin and Olshanski [10], we rewrite $\left(H(\lambda, 2) H^{\prime}(\lambda, 2)\right)^{-1}$ 
in a determinantal form

$$
\frac{1}{H(\lambda, 2) H^{\prime}(\lambda, 2)}=\operatorname{det}\left[\frac{1}{\left(\lambda_{j}-2 j+i+1\right) !}, \frac{1}{\left(\lambda_{j}-2 j+i\right) !}\right],
$$

where $1 \leq i \leq 2 l(\lambda), 1 \leq j \leq l(\lambda)$. Then the formula in the statement of the Proposition follows from the definition of $\mathcal{M}_{z, z^{\prime}, \theta, \xi}(\lambda)$ (equation $(2.2)$ ), and from equation (6.2).

Proposition 6.2. Let $\pi_{z}$ be the specialization of the algebra $\Lambda$ of symmetric functions defined by

$$
\pi_{z}\left(e_{k}\right)=\xi^{\frac{k}{2}} \frac{(z-k+1)(z-k+2) \ldots z}{k !}
$$

Then the following formula holds true

$$
\mathcal{M}_{z, z-1,2, \xi}(\lambda)=(1-\xi)^{\frac{z(z-1)}{2}} \operatorname{det}\left[\pi_{-z}\left\{e_{\lambda_{j}-2 j+i+1}\right\}, \pi_{-z}\left\{e_{\lambda_{j}-2 j+i}\right\}\right],
$$

where $1 \leq i \leq 2 l(\lambda)$, and $1 \leq j \leq l(\lambda)$.

Proof. Assume first that $z=N$. Then, using equation (6.3), we find that

$$
\pi_{z=N}\left\{e_{k}\right\}=\xi^{\frac{k}{2}} \frac{(N-k+1)(N-k+2) \ldots N}{k !}=\xi^{\frac{k}{2}}\left(\begin{array}{c}
N \\
k
\end{array}\right) .
$$

Let us compute the determinant

$$
\operatorname{det}\left[\pi_{z}\left\{e_{\lambda_{j}-2 j+i+1}\right\}, \pi_{z}\left\{e_{\lambda_{j}-2 j+i}\right\}\right],
$$

where $1 \leq i \leq 2 l(\lambda), 1 \leq j \leq l(\lambda)$, and the parameter $z$ equals $N$. Taking into account equation (6.4) we find

$$
\begin{aligned}
& \operatorname{det}\left[\pi_{z=N}\left\{e_{\lambda_{j}-2 j+i+1}\right\}, \pi_{z=N}\left\{e_{\lambda_{j}-2 j+i}\right\}\right] \\
& =\operatorname{det}\left[\xi^{\frac{\lambda_{j}-2 j+i+1}{2}}\left(\begin{array}{c}
N \\
\lambda_{j}-2 j+i+1
\end{array}\right), \xi^{\frac{\lambda_{j}-2 j+i}{2}}\left(\begin{array}{c}
N \\
\lambda_{j}-2 j+i
\end{array}\right)\right] \\
& =\xi^{|\lambda|} \frac{N !(N+1) !}{\left(N-\lambda_{1}\right) !\left(N-\lambda_{1}+1\right)} \frac{(N+2) !(N+3) !}{\left(N-\lambda_{2}+2\right) !\left(N-\lambda_{2}+3\right)} \times \ldots \\
& \times \frac{(N+2 l(\lambda)-2) !(N+2 l(\lambda)-1) !}{\left(N-\lambda_{l(\lambda)}+2 l(\lambda)-2\right) !\left(N-\lambda_{l(\lambda)}+2 l(\lambda)-1\right) !} \operatorname{det}\left[\frac{1}{\left(\lambda_{j}-2 j+i+1\right) !}, \frac{1}{\left(\lambda_{j}-2 j+i\right) !}\right] .
\end{aligned}
$$

This equation can be further rewritten as follows

$$
\begin{aligned}
& \operatorname{det}\left[\pi_{z=N}\left\{e_{\lambda_{j}-2 j+i+1}\right\}, \pi_{z=N}\left\{e_{\lambda_{j}-2 j+i}\right\}\right] \\
& =\xi^{|\lambda|} \prod_{i=1}^{l(\lambda)}(-N-2 i+2)_{\lambda_{i}}(-N-1-2 i+2)_{\lambda_{i}} \operatorname{det}\left[\frac{1}{\left(\lambda_{j}-2 j+i+1\right) !}, \frac{1}{\left(\lambda_{j}-2 j+i\right) !}\right]
\end{aligned}
$$


where $1 \leq i \leq 2 l(\lambda)$, and $1 \leq j \leq l(\lambda)$. As soon as $(z)_{\lambda, 2}=\prod_{i=1}^{l(\lambda)}(z-$ $2 i+2)_{\lambda_{i}}$, we see that the equation

$$
\begin{aligned}
& \operatorname{det}\left[\pi_{z}\left\{e_{\lambda_{j}-2 j+i+1}\right\}, \pi_{z}\left\{e_{\lambda_{j}-2 j+i}\right\}\right] \\
& =\xi^{|\lambda|}(-z)_{\lambda}(-z-1)_{\lambda} \operatorname{det}\left[\frac{1}{\left(\lambda_{j}-2 j+i+1\right) !}, \frac{1}{\left(\lambda_{j}-2 j+i\right) !}\right]
\end{aligned}
$$

holds true when $z=N$. By an analytic continuation, equation (6.5) also holds true for an arbitrary complex $z$. Comparing equations (6.1) and (6.5) we obtain the formula in the statement of the Proposition.

Now we are ready to complete the proof of Theorem 3.1. Proposition 6.2 implies that $\mathcal{M}_{\text {Schur,2 }}$ is reduced to $\mathcal{M}_{z, z-1,2, \xi}$ when the specialization $\pi$ in the definition of $\mathcal{M}_{\text {Schur }, 2}$ is $\pi_{-z}$. Therefore, the correlation function of $\mathcal{M}_{z, z-1,2, \xi}$ can be obtained from Theorem 4.1. We only need to compute $\pi_{-z}\{E(w)\}$, and $\pi_{-z}\left\{E\left(\frac{1}{w}\right)\right\}$. When $\pi=\pi_{z}$, where $\pi_{z}$ is the specialization of the algebra $\Lambda$ of symmetric functions defined by equation (6.3), we find

$$
\pi_{z}\{E(w)\}=\sum_{n=0}^{\infty}(-1)^{n} \frac{(-z)_{n}}{n !}(\sqrt{\xi} w)^{n}=(1+\sqrt{\xi} w)^{z},
$$

provided that the condition $|w|<\xi^{-1 / 2}$ is satisfied. We also obtain

$$
\pi_{z}\left\{E\left(\frac{1}{w}\right)\right\}=\sum_{n=0}^{\infty}(-1)^{n} \frac{(-z)_{n}}{n !}\left(\frac{\sqrt{\xi}}{w}\right)^{n}=\left(1+\frac{\sqrt{\xi}}{w}\right)^{z},
$$

where it is assumed that $|w|>\xi^{1 / 2}$. If $\xi^{1 / 2}<|w|<\xi^{-1 / 2}$ both equations (6.6) and (6.7) hold true. As $0<\xi<1$, we can choose the contours $\left\{w_{1}\right\},\left\{w_{2}\right\}$ as in Theorem 4.1, with the additional requirement that $\xi^{-1 / 2}$ lies outside both contours $\left\{w_{1}\right\}$ and $\left\{w_{2}\right\}$. This gives formulae in Theorem 3.1, a).

With the choice of the contour $\{w\}$ as in the statement of Theorem 3.1, the formula for the function $\Phi_{k}(x)$ (see equation (4.5)) takes the form

$$
\Phi_{k}(x)=\frac{1}{2 \pi i} \oint_{\{w\}}(1+\sqrt{\xi} w)^{-z}\left(1+\frac{\sqrt{\xi}}{w}\right)^{z} \frac{d w}{w^{k+x+1}} .
$$

The integral in the right-hand side of the formula above can be computed, and the function $\Phi_{k}(x)$ can be expressed as

$$
\Phi_{k}(x)=(-1)^{k+x} \xi^{\frac{k+x}{2}}(1-\xi)^{z}(z)_{k+x} \frac{F\left(-z+1,-z ; k+x+1 ; \frac{\xi}{\xi-1}\right)}{\Gamma(k+x+1)},
$$


where $F(a, b ; c ; u)$ denotes the Gauss hypergeometric function. Equation (4.4) gives the representation for the function $S_{z, z-1,2, \xi}(x, y)$ as in Theorem 3.1, b).

\section{The Pfaffian process Defined by the the Plancherel MEASURE WITH THE JACK PARAMETER $\theta=2$}

Theorem 7.1. a) For $\mathcal{M}_{\infty, \infty, 2, \eta}$ defined by equation (2.5) the point configurations $\mathcal{D}_{2}(\lambda)=\left\{\lambda_{i}-2 i\right\}_{i=1}^{\infty} \subset \mathbb{Z}$ form a Pfaffian point process. This means that the correlation function $\varrho_{\infty, \infty, 2, \eta}$ of $\mathcal{M}_{\infty, \infty, 2, \eta}$ defined by

$$
\varrho_{\infty, \infty, 2, \eta}=\mathcal{M}_{\infty, \infty, 2, \eta}\left(\left\{\lambda \mid X \subset \mathcal{D}_{2}(\lambda)\right\}\right),
$$

(where $X=\left(x_{1}, \ldots, x_{n}\right)$ is any fixed subset of $\mathbb{Z}$ consisting of $n$ distinct points) can be represented as a Pfaffian of a $2 \times 2$ matrix valued kernel. Namely,

$$
\varrho_{\infty, \infty, 2, \eta}=\operatorname{Pf}\left[K_{\infty, \infty, 2, \eta}\left(x_{i}, x_{j}\right)\right]_{i, j=1}^{n}
$$

where

$$
K_{\infty, \infty, 2, \eta}(x, y)=\left[\begin{array}{cc}
S_{\infty, \infty, 2, \eta}(x+1, y+1) & -S_{\infty, \infty, 2, \eta}(x+1, y) \\
-S_{\infty, \infty, 2, \eta}(x, y+1) & S_{\infty, \infty, 2, \eta}(x, y)
\end{array}\right]
$$

and

$$
\begin{aligned}
& S_{\infty, \infty, 2, \eta}(x, y) \\
& =\frac{1}{(2 \pi i)^{2}} \oint_{\left\{w_{1}\right\}} \oint_{\left\{w_{2}\right\}} \frac{d w_{1} d w_{2}}{w_{1}^{x} w_{2}^{y}} \exp \left[\sqrt{2} \eta\left(w_{1}-w_{1}^{-1}+w_{2}-w_{2}^{-1}\right)\right] \frac{\left(w_{2}-w_{1}\right)}{\left(w_{1} w_{2}-1\right)\left(w_{1}^{2}-1\right)\left(w_{2}^{2}-1\right)} .
\end{aligned}
$$

Here $\left\{w_{1}\right\},\left\{w_{2}\right\}$ are simple contours which both go around 0 in positive direction, and they are chosen in such a way that $\left|w_{1}\right|>1,\left|w_{2}\right|>1$.

b) The function $S_{\infty, \infty, 2, \eta}(x, y)$ can be written as

$$
S_{\infty, \infty, 2, \eta}(x, y)=\sum_{k, m=1}^{\infty} J_{k+x}(2 \sqrt{2} \eta)(\Upsilon)_{k, m} J_{m+x}(2 \sqrt{2} \eta)
$$

where $\Upsilon$ is defined by equation (3.1), and where $J_{\nu}(x)$ is the Bessel function, see Ref. [25], 7.2.4.

Proof. Consider the specialization $\pi_{\infty}^{(\sqrt{2} \eta)}$ of the algebra $\Lambda$ of symmetric functions defined by

$$
\pi_{\infty}^{(\sqrt{2} \eta)}\left(p_{k}\right)= \begin{cases}\eta, & k=1 \\ 0, & k=2,3, \ldots\end{cases}
$$


In this specialization we have

$$
\pi_{\infty}^{(\sqrt{2} \eta)}\left\{e_{k}\right\}=\frac{(\sqrt{2} \eta)^{k}}{k !}, \text { and } \pi_{\infty}^{(\sqrt{2} \eta)}\{E(w)\}=\exp (\sqrt{2} \eta w) .
$$

Next observe that $\mathcal{M}_{\text {Schur, } 2}$ is reduced to $\mathcal{M}_{\infty, \infty, 2, \eta}$, if the specialization $\pi$ in the definition of $\mathcal{M}_{\text {Schur,2 }}$ coincides with the specialization $\pi_{\infty}^{(\sqrt{2} \eta)}$. This follows from equations (4.1), and from the fact that

$$
\mathcal{M}_{\infty, \infty, 2, \eta}(\lambda)=e^{-\eta^{2}} \operatorname{det}\left[\frac{(\sqrt{2} \eta)^{\lambda_{j}-2 j+i+1}}{\left(\lambda_{j}-2 j+i+1\right) !}, \frac{(\sqrt{2} \eta)^{\lambda_{j}-2 j+i}}{\left(\lambda_{j}-2 j+i\right) !}\right],
$$

where $1 \leq i \leq 2 l(\lambda), 1 \leq j \leq l(\lambda)$. We then conclude that the formula for $\varrho_{\text {Plancherel,2 }}$ must have the same form as that for $\varrho_{\mathrm{Schur}, 2}$. As $\pi=\pi_{\text {Plancherel }}^{(\sqrt{2} \eta)}$ we check (taking into account equation (7.2)) that the formulae obtained in Theorem 4.1 are reduced to the formulae in the statement of Theorem 7.1 .

\section{REFERENCES}

[1] Baik, J.; Deift, P.; Johansson, K. On the distribution of the length of the longest increasing subsequence of random permutations. J. Amer. Math. Soc. 12 (1999), no. 4, 1119-1178.

[2] Baik, J.; Rains, E. M. The asymptotics of monotone subsequences of involutions. Duke Math. J. 109 (2001), no. 2, 205-281.

[3] Baik, J.; Rains, E. M. Symmetrized random permutations. Random matrix models and their applications, 1-19, Math. Sci. Res. Inst. Publ., 40, Cambridge Univ. Press, Cambridge, 2001.

[4] Baik, Jinho; Rains, Eric M. Algebraic aspects of increasing subsequences. Duke Math. J. 109 (2001), no. 1, 1-65.

[5] Borodin, A. Periodic Schur process and cylindric partitions. Duke Math. J. 140 (2007), no. 3, 391-468.

[6] Borodin, A.; Olshanski, G. Distributions on partitions, point processes, and the hypergeometric kernel. Comm. Math. Phys. 211 (2000), no. 2, 335-358.

[7] Borodin, A.; Okounkov, A.; Olshanski, G. Asymptotics of Plancherel measures for symmetric groups. J. Amer. Math. Soc. 13 (2000), no. 3, 481-515

[8] Borodin, A.; Olshanski, G. $z$-measures on partitions, Robinson-SchenstedKnuth correspondence, and $\beta=2$ random matrix ensembles. Random matrix models and their applications, 71-94, Math. Sci. Res. Inst. Publ., 40, Cambridge Univ. Press, Cambridge, 2001.

[9] Borodin, A.; Olshanski, G. Meixner polynomials and random partitions. Mosc. Math. J. 6 (2006), no. 4, 629-655, 771.

[10] Borodin, A.; Olshanski, G. Z-measures on partitions and their scaling limits. European J. Combin. 26 (2005), no. 6, 795-834.

[11] Borodin, A.; Olshanski, G. Harmonic functions on multiplicative graphs and interpolation polynomials. Electron. J. Combin. 7 (2000), Research Paper 28, $39 \mathrm{pp}$. 
[12] Borodin, A.; Olshanski, G.; Strahov, E. Giambelli compatible point processes. Adv. in Appl. Math. 37 (2006), no. 2, 209-248.

[13] Borodin, A.; Strahov, E. Averages of characteristic polynomials in random matrix theory. Comm. Pure Appl. Math. 59 (2006), no. 2, 161-253.

[14] Borodin, A.; Strahov, E. Correlation kernels for discrete symplectic and orthogonal ensembles. arXiv:0712.1693v2.

[15] Borodin, A.; Rains, E. M. Eynard-Mehta theorem, Schur process, and their Pfaffian analogs. J. Stat. Phys. 121 (2005), no. 3-4, 291-317.

[16] Deift, P. Four lectures on random matrix theory. Asymptotic combinatorics with applications to mathematical physics (St. Petersburg, 2001), 21-52, Lecture Notes in Math., 1815, Springer, Berlin, 2003.

[17] Deift, P. A. Orthogonal polynomials and random matrices: a Riemann-Hilbert approach. Courant Lecture Notes in Mathematics, 3. New York University, Courant Institute of Mathematical Sciences, New York; American Mathematical Society, Providence, RI, 1999.

[18] Ferrari, P. L. Polynuclear growth on a flat substrate and edge scaling of GOE eigenvalues. Comm. Math. Phys. 252 (2004), no. 1-3, 77-109.

[19] Ferrari, P. L.; Prhofer, M. One-dimensional stochastic growth and Gaussian ensembles of random matrices. Markov Process. Related Fields 12 (2006), no. 2, 203-234.

[20] Forrester, P.J. Log-gases and Random Matrices. http://www.ms.unimelb.edu.au/ matpjf/matpjf.html.

[21] Forrester, P.J.; Nagao, T.; Rains, E.M. Correlation functions for random involutions. Intern. Math. Res. Notices (2006) article ID 89796, 35 pages.

[22] Johansson, K. Toeplitz determinants, random growth and determinantal processes. Proceedings of the International Congress of Mathematicians, Vol. III (Beijing, 2002), 53-62, Higher Ed. Press, Beijing, 2002.

[23] Johansson, K. Non-intersecting paths, random tilings and random matrices. Probab. Theory Related Fields 123 (2002), no. 2, 225-280.

[24] Johansson, K. Random growth and random matrices. European Congress of Mathematics, Vol. I (Barcelona, 2000), 445-456, Progr. Math., 201, Birkhuser, Basel, 2001.

[25] Higher Transcendental functions, bateman Manuscript Project, McGraw-Hill, New York, 1953.

[26] Harnard, J.; Orlov, A.Yu. Fermionic construction of tau fanctions and random processes. arXiv:0704.1157v1.

[27] Kerov, S. Anisotropic Young diagrams and Jack symmetric functions. Funktsion. Anal. i. Prilozhen. 34 (1) (2000) 51-64 (in Russian).

[28] Kerov, S.; Olshanski, G.; Vershik, A. Harmonic analysis on the infinite symmetric group. Invent. Math. 158 (2004), no. 3, 551-642.

[29] Macdonald, I.G. Symmetric functions and Hall polynomials. Second Edition, Oxford University Press, 1995.

[30] Mehta, M. L. Random Matrices. 2nd ed. San Diego: Academic, 1991.

[31] van Moerbeke, P. Random and Integrable Models in Mathematics and Physics. arXiv:0712.3847.

[32] Okounkov, A. Infinite wedge and random partitions. Selecta Math. (N.S.) 7 (2001), no. 1, 57-81. 
[33] Okounkov, A. Symmetric functions and random partitions. Symmetric functions 2001: surveys of developments and perspectives, 223-252, NATO Sci. Ser. II Math. Phys. Chem., 74, Kluwer Acad. Publ., Dordrecht, 2002.

[34] Okounkov, A. The uses of random partitions. XIVth International Congress on Mathematical Physics, 379-403, World Sci. Publ., Hackensack, NJ, 2005.

[35] Okounkov, A. SL(2) and $z$-measures. Random matrix models and their applications, 407-420, Math. Sci. Res. Inst. Publ., 40, Cambridge Univ. Press, Cambridge, 2001.

[36] Okounkov, A.; Reshetikhin, N. Correlation function of Schur process with application to local geometry of a random 3-dimensional Young diagram. J. Amer. Math. Soc. 16 (2003), no. 3, 581-603

[37] Olshanski, G. An introduction to harmonic analysis on the infinite symmetric group. Asymptotic combinatorics with applications to mathematical physics (St. Petersburg, 2001), 127-160, Lecture Notes in Math., 1815, Springer, Berlin, 2003.

[38] Prhofer, M.; Spohn, H. Statistical self-similarity of one-dimensional growth processes. Statistical mechanics: from rigorous results to applications. Phys. A 279 (2000), no. 1-4, 342-352.

[39] Rains, E. Correlation functions for symmetrized increasing subsequences. arXiv:math $/ 0006097 \mathrm{v} 1$

[40] Sasamoto, T.; Imamura, T. Fluctuations of the one-dimensional polynuclear growth model in half-space. J. Stat. Phys. 115 (2004), 749-803.

[41] Soshnikov, A. Determinantal random point fields. (Russian) Uspekhi Mat. Nauk 55 (2000), no. 5(335), 107-160; translation in Russian Math. Surveys 55 (2000), no. 5, 923-975.

[42] Tracy, C.A.; Widom, H. Correlation functions, cluster functions and spacing distributions for random matrices. J. Stat. Phys. 96 (1998) 809-835.

[43] Vuletić, M . Shifted Schur process and asymptotics of large random strict plane partitions. Intern. Math. Res. Notices (2007) article ID rnm043, 53 pages. 